\title{
Laboratory diagnosis of Pneumocystis carinii pneumonia
}

The role of the laboratory in the diagnosis of any infection depends on the clinical circumstances. Pneumocystis carinii pneumonia (PCP) is an excellent example of this. When patients with human immunodeficiency virus (HIV) infection present late in their illness with pneumonia, the clinical presentation alone can be diagnostic and laboratory diagnosis of PCP may be unnecessary. However, in patients with early disease, including those who may be receiving prophylactic chemotherapy, or those who are not obviously immunocompromised, the laboratory diagnosis of PCP is critical for correct management.

PCP can be confirmed only by laboratory tests and current dogma is that these require respiratory samples obtained by invasive procedures. ${ }^{1,2}$ The fact that such procedures may not be possible in very ill patients has stimulated interest in a plethora of non-invasive methods, including various exercise-induced hypoxaemia tests, pulmonary function tests, gallium scanning and radiolabelled technetium diethylenetriamine penta-acetate (DTPA) clearance tests. ${ }^{1,2}$ To complicate matters, some workers have postulated a scoring system to diagnose $\mathrm{PCP},{ }^{3}$ and others have suggested a diagnostic algorithm. ${ }^{4}$ There is a danger that investigation may become over-complicated, taking diagnosis in the wrong direction. All non-invasive procedures are non-specific and there is a good case for developing definitive laboratory tests that require less invasive specimens.

$\dot{A}$ decade ago, $P$. carinii was regarded as an unusual protozoon infecting immunocompromised individuals. Now there is evidence that the organism should be reclassified as a fungus and the infection is being recognised in individuals who are not obviously immunocompromised, such as the elderly. ${ }^{5}$ The fungal classification has important implications as it suggests that the organism might be widespread and have other stages, such as spores, which have not yet been identified. ${ }^{5}$ The existence of spores would give a new dimension to our understanding of this infection. There is also greater recognition of the relative importance of the two stages of the organism that have been identified already. Laboratory tests tend to concentrate on the more easily identified cyst stage, but may be more profitably targeted at the trophozoites which are much more common in infection. ${ }^{6}$ Trophozoites are more fragile than cysts and care must be taken not to destroy them during the sample preparation procedure. ${ }^{6}$ They are also morphologically indistinct and, consequently, difficult to identify reliably by staining. They are more easily recognised by some immunofluorescent monoclonal antibody methods, ${ }^{1}$ or by the polymerase chain reaction (PCR), which will identify DNA from any stage of $P$. carinii in the sample. ${ }^{7}$ Serology may also detect antibodies against trophozoites provided that they are included in the antigen preparation. ${ }^{6}$

It is most important that clinical and laboratory personnel are fully aware of the clinical spectrum of pneumocystosis, especially in those who are not obviously immunocompromised.

Pneumocystosis is a form of atypical pneumonia and the same approach to diagnosis should be taken. Diagnosis should not be left to specialised units or laboratories; all hospitals with acute medical services must be clear on their approach to this infection. In immunocompromised patients with pneumonia, especially those with HIV infection, PCP should be the first consideration; in other patients, PCP should be considered only after initial management has been unsuccessful. As with other atypical pneumonias, additional samples may reveal the cause and serology may be helpful, especially as serum samples are obtained easily, even from very ill patients. Standard immunofluorescence and ELISA tests detect IgG antibodies to $P$. carinii. The present tests have poor sensitivity and specificity, so that significant rises in titre provide the best evidence of current infection, ${ }^{1,8}$ although a raised titre in a single specimen tested by a method with low sensitivity but better specificity can also be significant. However, serology is less useful in immunosuppressed patients, particularly those with HIV infection or AIDS. ${ }^{8}$ Other antibody class responses in PCP have been investigated but are generally considered unhelpful.

There has been too much emphasis on esoteric tests that are not available in most hospitals and, therefore, do not address adequately the problem of PCP diagnosis. They all require a high level of interpretative skills. Radiolabelled scanning methods are slow (2-3 days for a gallium scan result) and unsuited to rapid screening of high risk individuals. ${ }^{4}$ Some are as impracticable in very ill patients as the collection of bronchoalveolar lavage or induced sputa.

Our approach is more pragmatic. All hospitals should have access to methods for the detection of pneumocystis cysts in bronchoalveolar lavage samples or induced sputum. Immunofluorescent monoclonal antibody detection methods applied to deep lung secretions have a very high sensitivity, even in patients receiving aerosolised pentamidine prophylaxis with breakthrough infection. ${ }^{9}$ Samples are collected preferably by fibre-optic bronchoscopy, from multiple lobes if necessary. In patients who are too ill for these 
investigations, sputum should be examined and serum sent to more specialised laboratories for serological tests, as is done for fungal and some other atypical pneumonias. Evidence is accumulating that PCR is sensitive enough to enable a diagnosis to be made from samples acquired by less invasive means. ${ }^{7,10}$ Apart from sputum, investigation of nasopharyngeal secretions should be considered. This may be particularly appropriate for acutely ill children, in whom sputum induction is not practicable. ${ }^{11}$ The problem with examining non-invasive specimens by PCR is that a positive result may indicate asymptomatic infection rather than overt disease. ${ }^{1,2}$ However, if positive results are also accompanied by positive serological results, it is likely that they are indicative of active disease. $^{1}$

This is a technological age and there is a great temptation to make life more complicated than it

\section{References}

1. Chatterton JMW, Ho-Yen DO. Laboratory investigation of Pneumocystis carinii pneumonia. Genitourin Med 1992; 68 336-341.

2. Malin AS, Miller RF. Pneumocystis carinii pneumonia; presentation and diagnosis. Rev Med Microbiol 1992; 3: 80-87.

3. Smith D, Forbes A, Gazzard B. A simple scoring system to diagnose Pneumocystis carinii pneumonia in high-risk individuals. AIDS $1992 ; 6: 337-338$.

4. Smith DE. Efficient diagnosis of Pneumocystis carinii pneumonia. Int J STD AIDS 1994; 5: 1-7.

5. Walzer PD. Pneumocystis carinii-new clinical spectrum? $N$ Engl J Med 1991; 324: 263-265.

6. Chatterton JMW, Joss AWL, Davidson MM, Ho-Yen DO Why have Pneumocystis carinii trophozoites been ignored? $J$ Clin Pathol 1990; 43: 265-268.

7. Evans R, Joss AWL, Pennington TH, Ho-Yen DO. The use of nested polymerase chain reaction for detecting Pneumocystis carinii from lung and blood in rat and human infection. J Med Microbiol 1995; 42: 209-213 needs to be. Yet, problems of the past are similar to problems of the future. PCP is both a fungal infection and an atypical pneumonia which can be investigated by techniques that are now routine in most hospitals in the developed world. Later in the illness, especially if there are complications, further tests performed in specialised laboratories may be required. Pneumocystosis should no longer be regarded as esoteric, but rather as an infection whose initial diagnosis should be within the scope of all clinical microbiology laboratories.

D. O. Ho-Yen,

J. M. W. Chatterton and

A. W. L. Joss

Microbiology Department, Raigmore Hospital NHS Trust, Inverness IV2 3UJ

8. Elvin K, Björkman A, Heurlin N, Eriksson B-M, Barkholt L, Linder E. Seroreactivity to Pneumocystis carinii in patients with AIDS versus other immunosuppressed patients. Scand $J$ Infect Dis 1994; 26: 33-40.

9. Levine SJ, Kennedy D, Shelhamer JH et al. Diagnosis of Pneumocystis carinii pneumonia by multiple lobe, sitedirected bronchoalveolar lavage with immunofluorescent monoclonal antibody staining in human immunodeficiency virus-infected patients receiving aerosolized pentamidine chemoprophylaxis. Am Rev Respir Dis 1992; 146: 838-843.

10. Lipschik GY, Gill VJ, Lundgren JD et al. Improved diagnosis of Pneumocystis carinii infection by polymerase chain reaction on induced sputum and blood. Lancet $1992 ; 340$ : 203-206.

11. Hague RA, Burns SE, Mok JYQ, Yap PL. Diagnosis of Pneumocystis carinii pneumonia from non-invasive sampling of respiratory secretions. Arch Dis Child 1990; 65: 1364-1365. 